\title{
LASER LINEAR COLLIDER WITH A TRAVELLING LASER FOCUS SUPPLY
}

\author{
A. A. Mikhailichenko* \\ Wilson Laboratory, Cornell University, Ithaca, NY 14850
}

\begin{abstract}
Requirements considered for $2 \times 1 \mathrm{~km}$ long linac, driven by a laser radiation distributed within open accelerating structure with special sweeping devices. These devices deflect the laser radiation to the open accelerating structure in accordance with instant position of accelerated particles The power reduction and shortening the time of illumination of every point of the structure is equal to the number of resolved spots, associated with the sweeping device. A $300 \mathrm{~J}, 100$-ps laser flash could provide the energy $30 \mathrm{TeV}$ for $\lambda \cong 1 \mu \mathrm{m}$ and $3 \mathrm{TeV}$ for $\lambda \cong 10 \mu \mathrm{m}$ on $1 \mathrm{~km}$ with the method described. For repetition rate $160 \mathrm{~Hz}$ the luminosity associated with colliding beams could reach $10^{33} \mathrm{~cm}^{-2} \mathrm{~s}^{-1}$ per bunch. Wall plug power required for operation of LLC is $\sim 2 \mathrm{MW}$.
\end{abstract}

\section{INTRODUCTION}

The concept of a Linac, driven by a Traveling Laser Focus (TLF) method was originated in [1-3]. TLF deals with accelerating structure, what scaled down to a laser wavelength. The beam is going inside the structure similar to the existing practice. Tiny dimensions of the beam required could be achieved by proper preparation of the beam [4]. For excitation of accelerating cells, each of them has an opening from one side, what gives a possibility for parallel excitation. Laser radiation focused onto these openings. So the cells excited locally. Focusing in transverse direction is more radical, as the focusing lens could be positioned very close to the accelerating structure. Special sweeping (deflecting) device moves this focal spot in longitudinal direction in accordance with instant position of the particle. Due to this arrangement, all impulse laser power is acting for generation of accelerating field at the instant particle's location only. These arrangements reduce the power required from the source (laser) and reduce illuminating time. It was shown [2], that the power reduction and shortening of illuminating time of any point on the surface equal numerically to the number of resolved spots (pixels), associated with the sweeping device. One can see that the cells are working with zero phase shift per cell what yields the distance between the cells equal to the laser wavelength. The number of the particles required is about one million only, so the problems with wakes here are not more complicated, than in usual $\mathrm{cm}$-wavelength structure.

\section{TLF METHOD}

Fig. 1 reminds the idea [1-3]. The laser bunch with longitudinal dimension much bigger, than transverse ones passing trough the sweeping device. This fast sweeping device is driven by electrical pulse synchronously with the particle's passage. It directs the beginning of the laser bunch to the entrance of accelerating structure and the end-to the exit of the structure. So closer to the structure laser bunch has an instant slope of 45 degrees to the direction of sweeping device. The local wave fronts still rectangular to the sweeping device, however. All deflecting elements are synchronized so that the electron bunch has continuous acceleration in all sections. One can see from Fig. 1, that the energy concentrated in the laser's bunch volume delivered here by the source by the time defined by the length of the laser bunch what is equal to the length of accelerating structure in Fig.1.

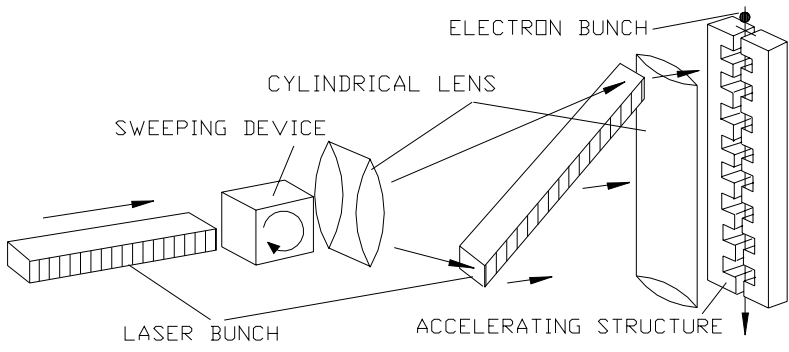

Figure 1: The principle. Lines across the laser bunch indicate the direction of wave fronts.

Optimal laser focus sweep is about $3 \mathrm{~cm}$ for a practical reasons, so the accelerating device looks like a sequence of $3 \mathrm{~cm}$ - long accelerating structures. A short focusing cylindrical lens installed close to accelerating structure reduces the transverse size of the spot to a minimal one. Each part of the grating structure is illuminated by duration, which is defined by time $\tau \cong L / N_{R} c \cong 100 \lambda$, where $N_{R}$-is the number of resolved spots, (see lower), $L-$ is the length of the structure. For $\lambda=1 \mu \mathrm{m}$, then $\tau \cong 3 \cdot 10^{-13} \mathrm{sec}$. This time is less than the time between electron-electron collisions, what is $\tau_{\text {coll }} \cong 10^{-12} \mathrm{sec}$, still however longer, than the time, corresponding to reaction of electron plasma in a metal, what is $\tau_{p} \approx 3 \cdot 10^{-16} \mathrm{~s}$. TLF method does not exclude the problems, associated with a breakdown limit of the structure, but it allows drastically reduce the laser impulse power, required for excitation the structure to this level. The latest measurements show a damage threshold increase while the illumination time is shortening. This breaks the $\tau^{1 / 2}$ low. This was explained by saturation of impact

\footnotetext{
${ }^{*}$ Work supported by NSF. Phone: (607) 255-3785, Fax: (607) 255-8062, e-mail "mikhail@1ns62.lns.cornell.edu
} 
ionization rate per unit distance [5]. Measured threshold for 0.' ps pulse was about $10 \mathrm{~J} / \mathrm{cm}^{2}$. For 1 ps the threshold measurer was $6 \mathrm{~J} / \mathrm{cm}^{2}$. In our case we have $10 \mathrm{~mJ}$ laser pulse distributer over area $3 \times 10^{-3} \mathrm{~cm}^{2}$ what gives $3 \mathrm{~J} / \mathrm{cm}^{2}$ and acceleratin! gradient $3 \mathrm{GeV} / \mathrm{m}$. We believe that these figures could $b_{1}$ improved after careful preparation the surface and optimizin! the working temperature. Main components of the schem considered below.

\section{DEFLECTINGDEVICE}

The ratio of deflection angle $\vartheta$ to diffraction angle $\vartheta_{d} \cong \lambda / a$, where $a$ is an aperture of the deflecting device, defines the number of resolved spots (pixels), $N_{R}=\vartheta / \vartheta_{d}$. The number of resolved spots $N_{R}$ is an invariant under any optical transformations. As we mentioned above, $N_{R}$ value gives the number for the lowering the laser power for gradient desired and, also, the number for the duty time reduction.

Electro-optical devices use controllable dependence of refractive index on electrical field strength and direction applied to some crystals (see Ref. To [2-3]). Short laser bunch could be swept with a travelling wave sweeping device [3], Fig. 2 below.

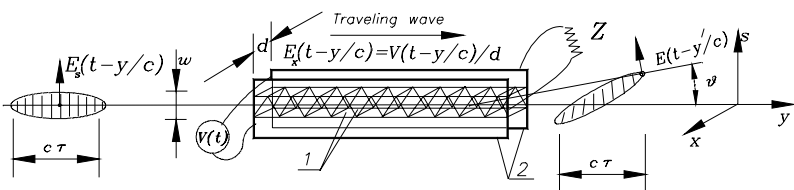

Figure 2: Deflecting device principle. Prisms 1 with oppositely directed optical axes installed in series between two parallel strip-line electrodes $2, d$-is the distance between them. $Z-$ is a matching impedance.

Lines across the laser bunch schematically show the wave fronts. $E_{x}(t-y / c)$-is a driving electrical field.

For the length of deflecting device $L=50 \mathrm{~cm}$, one can expect $\quad \Delta \vartheta \cong 10^{-2} \mathrm{rad} . N_{R} \cong 10$ for $\lambda \cong 10 \mu \mathrm{m}$ and $N_{R} \cong 100$ for $\lambda \cong 1 \mu \mathrm{m}$. Mechanical deflection system and Acousto-optical deflector could be also used here [3]. Basically they give the same deflecting parameters.

\section{ACCELERATING STRUCTURE}

We took the foxhole-type structure described in [6] as a basis, Fig. 3. This structure has an advantage in pumping possibilities. The structure gives a good positioning for electrical field map [2,3].

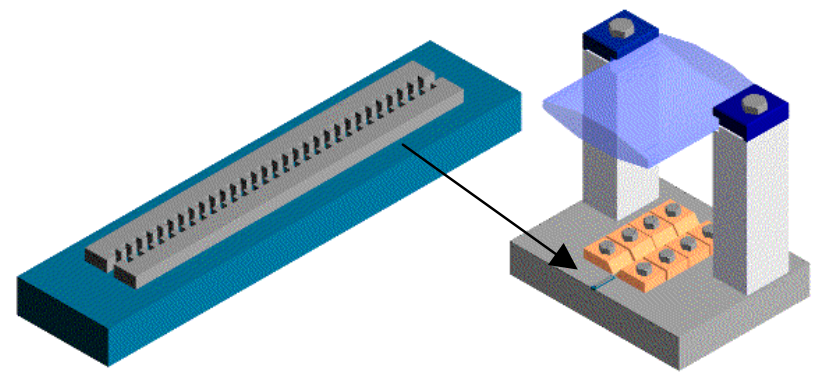

Figure 3: Accelerating structure and cylindrical lens. Structure on the left is scaled. Covers on accelerating structure are not shown.

Covers adjust the coupling between the groove and outer space. With these covers the height of a structure is about half of a laser wavelength and the cells have inductive coupling with outer space. The $Q_{R F}$ factor of the order 510 could be expected here. Technological possibilities in lithography allow a structure fabrication for a laser with a wavelength $\lambda \cong 1 \mu \mathrm{m}$. Each structure installed on a microtable moved by a piezoelectric. Calculations for this type of structure done with GdfidL code [7]. There were investigated different shapes of cell and transit slit between the cells. Calculation shows that wakes are acceptable here. Despite dimensions are small, the bunch population about $10^{6}$ only drastically rejects the wake problems.

\section{DESCRIPTION OF LLC}

Parameters of this LLC are represented in Table 1. The beams of electrons and positrons can be polarized what gives the effective gain in luminosity and reduces the background. Details one can find in $[2,3]$.

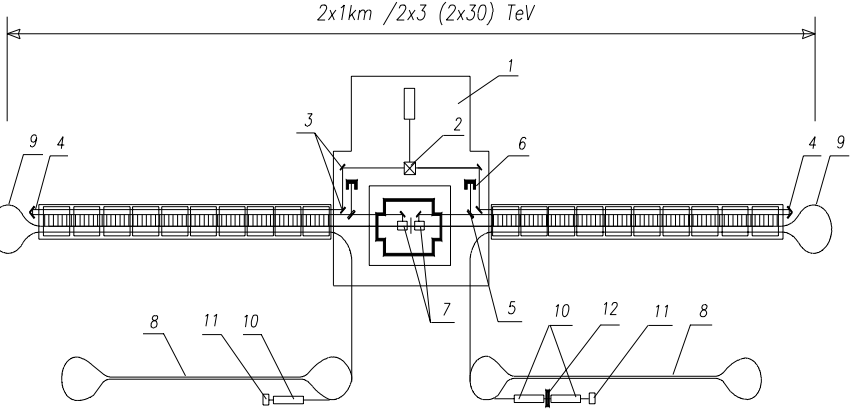

Figure 4: Linear Laser Collider (LLC) complex. 1-is a laser master oscillator platform, 2 -is an optical splitter, 3,4-are the mirrors, 5-is a semi-transparent mirror, 6-is a light absorber. 7-are the Final Focus Systems. 8-are the damping systems for preparing particle's beams with small emittances, 9-are the bends for particle's beam. 10-are the accelerating X-band structures, 11-is electron gun (polarized), 12- is a positron converter. 
TABLE 1. Parameters of Laser Linear Collider.

\begin{tabular}{ccc}
\hline Parameter & $\lambda \cong 10 \mu \mathrm{m}$ & $\lambda \cong 1 \mu \mathrm{m}$ \\
\hline Energy of $e^{ \pm}$beam & $3 \times 3 \mathrm{TeV}$ & $30 \times 30 \mathrm{TeV}$ \\
Length of section & $3 \mathrm{~cm}$ & $3 \mathrm{~cm}$ \\
Total length & $2 \times 1 \mathrm{~km}$ & $2 \times 1 \mathrm{~km}$ \\
Main linac gradient & $3 \mathrm{GeV} / \mathrm{m}$ & $30 \mathrm{GeV} / \mathrm{m}$ \\
Luminosity/bunch & $\leq 1 \cdot 10^{33} \mathrm{~cm}^{-2} \mathrm{~s}^{-1}$ & $\leq 1 \cdot 10^{33} \mathrm{~cm}^{-2} \mathrm{~s}^{-1}$ \\
Bunches/pulse & $3(\leq 100)^{*}$ & $10(\leq 300)^{*}$ \\
Laser flash J/Linac & 300 & 300 \\
Repetition rate & $160 \mathrm{~Hz}$ & $160 \mathrm{~Hz}$ \\
Beam power/Linac & $2.3 \mathrm{~kW}$ & $760 \mathrm{~W}$ \\
Bunch population & $3 \cdot 10^{7}$ & $10^{6}$ \\
Bunch length & $1 \mu \mathrm{m}$ & $0.1 \mu \mathrm{m}$ \\
$\gamma \varepsilon_{x} / \gamma \varepsilon_{y}$ cmrad & $10^{-8} / 4 \cdot 10^{-10}$ & $5 \cdot 10^{-9} / 1 \cdot 10^{-10}$ \\
Damping ring energy & $0.7 \mathrm{GeV}$ & $0.7 \mathrm{GeV}$ \\
Wall plug power** & $2 \times 0.5 \mathrm{MW}$ & $2 \times 0.5 \mathrm{MW}$ \\
\hline
\end{tabular}

*-Maximal possible number.

$* *$ Laser efficiency $\approx 10 \%$

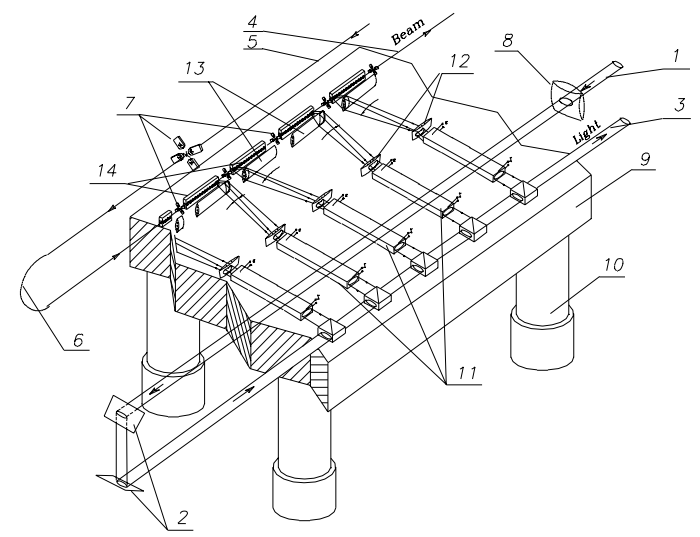

Figure 5: Table with a regular section of accelerator. 1,3is a laser beam. 4,5-the beam of particles. 7 and 8-are the focusing elements for the particle's and laser beam correspondingly. 9 -is an optical platform. Legs 10 have active damping system to minimize vibrations. 11-are the sweeping devices 12-are the lenses for focusing the laser radiation in longitudinal direction. 13-are short focusing cylindrical lenses. 14-are accelerating structures

After the bend in systems 2,6 on the back way to IP necessary voltages applied to correcting elements, distributed along the linac.

A kayak-paddle cooler pos. 8, Fig.4 serves as injector [4]. This is basically a racetrack, which has a sequence of wigglers and RF cavities in a straight section. This injector yields the equilibrium emittances

$$
\left(\gamma \varepsilon_{x}\right) \cong 1 \cdot 10^{-8} \mathrm{~cm} \cdot \mathrm{rad},\left(\gamma \varepsilon_{y}\right) \cong 4 \cdot 10^{-10} \mathrm{~cm} \cdot \mathrm{rad} .
$$

Scrapping extra particles remains a valid procedure for the emittance lowering. Intra-Beam Scattering is reduced for diluted beam. The focusing system includes the quadrupole lenses of appropriate dimensions and a $\mathrm{RF}$ focusing. Accelerating gradient defined by the laser flash energy $Q$. A laser flash with
$Q \cong 10 \mathrm{~mJ}, \tau \cong 0.1 \mathrm{~ns}$ at $\lambda \cong 1 \mu \mathrm{m}$ is able to feed the accelerating structure with the length about $c \tau \approx 3 \mathrm{~cm}$ with $\geq 30 \mathrm{GeV} / \mathrm{m}$. Nd-Glass laser can be used here. $\mathrm{CO}_{2}$ laser based system gives lower final energy. The power required $P_{i} \cong Q / \tau \approx 10^{8} \mathrm{~W}$ is within routinely obtained. The laser amplifier could be sectioned with few structures or even each $3 \mathrm{~cm}$ section could has it's own amplifier. For pumping the driving lasers the diode laser arrays could be used for the wavelengths indicated.

Bunch population defined by a loading of structure and by wakes. With $5 \%$ loading $N \cong 1 \cdot 10^{6}$ for $\lambda \cong 1 \mu \mathrm{m}$.

For $\lambda \cong 10 \mu \mathrm{m}$ this will be $N \cong 3 \cdot 10^{7}$. Final focus made with a multiplet of FODO structures having the number of the RF lenses in it of the order of few hundreds. Luminosity could reach $L \approx 1 \cdot 10^{33} \mathrm{~cm}^{-}{ }^{2} \mathrm{~s}^{-1}$ for $\lambda \cong 10 \mu \mathrm{m}, \gamma=2 \cdot 10^{6}(p c=1 \mathrm{TeV}), N \cong 2 \cdot 10^{7}, f \cong 160$ Hz. For $\lambda \cong 1 \mu \mathrm{m}$ result will be about the same.

\section{CONCLUSION}

Reduction of illuminating time and total laser power (or the flash energy) both defined by the number of resolved spots (pixels) associated with a deflecting device. Number of resolved spots $\approx 20-100$ achievable. Lasers for the TLF method need to have more power in intermediate time duration $\tau \approx 100 \mathrm{ps}$ rather than in a shorter time interval. Equivalent time of illumination of accelerating structure with this pulse is $0.1 \div 1 \mathrm{ps}$, however. For a $0.5 \times 0.5 \mathrm{TeV}$ Collider the length becomes $2 \times 170 \mathrm{~m}$ for $\lambda \cong 10 \mu \mathrm{m}$ and $2 \times 17 \mathrm{~m}$ for $\lambda \cong 1 \mu \mathrm{m}$, respectively.

\section{REFERENCES}

[1] A.A. Mikhailichenko, The method of acceleration of charged particles, Author's certificate USSR $\mathrm{N}^{\circ}$ 1609423, Priority May 1989, Bulletin of Inventions (in Russian), N6, p.220, 1994.

[2] A.A. Mikhailichenko, A concept of a Linac Driven by a Traveling Laser Focus, $7^{\text {th }}$ Advanced Accelerator oncepts Workshop, AIP 398 Proceedings, p.547.

[3]A.A. Mikhailichenko, Laser Acceleration: a Practical Approach, CLNS 97/1529, Cornell, 1997; also a Talk on LASERS'97, Fairmont Hotel, New Orleans LA, December 15-19, 1997.

[4] A.A. Mikhailichenko, Injector for a Laser Linear Collider, CLNS 98/1668.

[5] D. Du, X. Lu, G. Korn, J Squuier, G. Mourou, Optical Breackdown with Femtosecond Laser Pulses, CLEO94, May 8-13, 1994, Anaheim, California, Vol.8, p.407.

[6] R.C.Fernow, J.Claus, AIP Conference Proceedings, 279, 1992, p.212.

[7] W. Bruns, GdfidL, TU Berlin, 1997. 